\title{
Leukopenia is a biomarker for effective temozolomide dosing and predicts overall survival of patients with glioblastoma
}

\author{
K. GRACE HO ${ }^{1}$, ERIK N. UHLMANN ${ }^{2}$, ERIC T. WONG ${ }^{1}$ and ERIK J. UHLMANN ${ }^{1}$ \\ ${ }^{1}$ Department of Neurology, Beth Israel Deaconess Hospital and Harvard Medical School, Boston, MA 02215; \\ ${ }^{2}$ Khoury College of Computer Sciences, Northeastern University, Boston, MA 02115, USA
}

Received January 15, 2020; Accepted September 17, 2020

DOI: $10.3892 / \mathrm{mco} .2020 .2150$

\begin{abstract}
The median survival time of patients with glioblastoma is 14-16 months with a 5-year overall survival rate of $9.8 \%$. Standard of care treatment includes radiation with concomitant temozolomide followed by cyclic temozolomide. If the patient develops myelosuppression (thrombocytopenia, leukopenia or anemia), the dose of temozolomide is reduced or stopped to avoid bleeding or infections. Recent studies have demonstrated that mild leukopenia is associated with increased overall survival in patients with glioblastoma. To confirm prior results showing that leukopenia is associated with increased overall survival as a primary outcome in patients with glioblastoma, the present study retrospectively collected complete blood counts from 141 patients with glioblastoma treated at the Beth Israel Deaconess Medical Center (Boston, USA) between January 2012 and December 2017. According to Kaplan-Meier analysis with a log-rank test, the presence of leukopenia was associated with increased overall survival $(\mathrm{P}=0.008)$. Furthermore, patients with grade 2 leukopenia (Common Terminology Criteria for Adverse Events version 5.0) survived longer than those without myelosuppression $(\mathrm{P}=0.024)$. There was no difference in overall survival between patients with grade 1,3 or 4 leukopenia and those without myelosuppression. Leukopenia was associated with longer survival independent of age or extent of surgery in Cox proportional hazards regression modeling $(\mathrm{P}=0.00205)$. A possible interpretation is that grade 2 leukopenia is a biomarker of adequate temozolomide
\end{abstract}

Correspondence to: Dr Erik J. Uhlmann, Department of Neurology, Beth Israel Deaconess Hospital and Harvard Medical School, 330 Brookline Avenue, Boston, MA 02215, USA

E-mail: euhlmann@bidmc.harvard.edu

Abbreviations: GBM, glioblastoma; IMRT, intensity-modulated radiation therapy; TMZ, temozolomide; MGMT, $\mathrm{O}^{6}$-methylguanineDNA methyltransferase; CTCAE, Common Terminology Criteria for Adverse Events; PFS, progression-free survival; OS, overall survival; WBC, white blood cell count; IDH1, isocitrate dehydrogenase 1; EGFR, epidermal growth factor receptor

Key words: glioblastoma, bone marrow suppression, temozolomide, biomarker, survival dosing in a population with diverse DNA repair function, which may be the consequence of variable $\mathrm{O}^{6}$-methylguanine-DNA methyltransferase activity. A prospective dose escalation trial is necessary to determine if treatment-induced leukopenia is beneficial for all patients receiving temozolomide.

\section{Introduction}

Glioblastoma (GBM) is the most common adult malignant brain tumor and has a uniquely poor prognosis. Median survival is 14-16 months, and overall survival (OS) at 2 years is 27.2 and $9.8 \%$ at 5 years (1-3). Standard of care treatment consists of maximal safe resection of the enhancing portion of the tumor followed by 30 times 2 Gray daily fractionated intensity-modulated radiation therapy (IMRT) with concomitant temozolomide (TMZ) dosed at $75 \mathrm{mg} / \mathrm{m}^{2} /$ day. This is followed by at least six cycles of five daily doses of TMZ $\left(150 \mathrm{mg} / \mathrm{m}^{2}\right.$ for the first month and $200 \mathrm{mg} / \mathrm{m}^{2}$ for subsequent treatments) with each cycle being 28 days long (3).

Temozolomide is an oral alkylating agent that leads to the formation of specific DNA adducts almost exclusively repaired by $\mathrm{O}^{6}$-methylguanine-DNA methyltransferase (MGMT) (4). In rapidly dividing cells, there is less time for the repair to take place, allowing for selective damage or killing of GBM cells (5). GBM tumor cells and somatic cells, including bone marrow progenitors, largely share MGMT polymorphisms and promoter methylation status. The limiting toxicity of TMZ is myelosuppression (particularly thrombocytopenia) and is thought to be due to low MGMT activity in hematopoietic progenitors (6). If severe myelosuppression occurs, the current practice is to stop or reduce the dose of TMZ to limit the risk of complications and allow for marrow recovery. For efficient treatment, however, the dose of TMZ should be adjusted according to individual MGMT activities (7). MGMT activity assays are available for research but not for routine clinical use. A surrogate marker of somatic MGMT repair activity during TMZ treatment may be myelosuppression.

Studies using various chemotherapies other than TMZ have suggested that greater toxicity to certain organ systems may reflect increased potency of the drug and is therefore associated with better treatment effect, including longer progression-free survival (PFS) (8-10). Recent reports suggest that decreases in platelet count and white blood cell count in the setting of TMZ use is associated with increased OS 
in GBM patients (11-13). Our objective is to reproduce this finding and verify whether leukopenia in the setting of TMZ use is associated with increased OS in GBM patients at our institution.

\section{Materials and methods}

Patients. To be included in the study, patients must have histologically confirmed GBM and exposure to TMZ. Baseline and follow-up complete blood counts (CBC) were stored in the Beth Israel Deaconess Medical Center's electronic medical record system as part of routine clinical care. Patients were not excluded for any other reason such as demographics, clinical or radiologic outcomes. One hundred and forty-one patients met inclusion criteria. Age, sex, extent of surgical resection (categorized as biopsy, partial resection, and complete resection), radiation dose, and chemotherapy treatments were recorded. CBCs were recorded at baseline (defined as the most recent set of laboratory results prior to TMZ exposure) and at various time points available throughout the patient's course of TMZ up to six cycles of adjuvant TMZ. The primary endpoint was OS, and the secondary end point was PFS. This study was reviewed and approved by the Institutional Review Board of Beth Israel Deaconess Medical Center (Boston, MA). Patients were retrospectively identified using a database of GBM patients seen at Beth Israel Deaconess Medical Center between January 2012 and December 2017.

Data collection and statistical analysis. The severity of leukopenia was classified based on the National Cancer Institute's Common Terminology Criteria for Adverse Events (CTCAE version 5.0). OS was measured from the date of tissue diagnosis to the date of death due to any cause. Patients who were still alive by July 2020 were censored. Kaplan-Meier analysis with log rank and Cox multivariate proportional hazards analysis were performed using statistical computing language and survival analysis libraries ( $\mathrm{R}$ version 4.0.2, $\mathrm{R}$ Foundation, Vienna, Austria; 'survival' library version 3.2-3; 'survminer' library version 0.4 .8 ). Individual de-identified participant data used in this publication, including patient age, sex, extent of surgery, length of TMZ treatment, radiation dose, time to progression, time to death, baseline WBC and nadir WBC is available as supplementary material for download (Table SI).

\section{Results}

Patient characteristics. Between January 2012 and December 2017, 141 biopsy-proven GBM patients exposed to TMZ were retrospectively identified. Patient characteristics were comparable with respect to sex, extent of surgical resection, and fraction of patients completed six weeks of IMRT/TMZ followed by six cycles of adjuvant TMZ (Table I). The BIDMC cohort was older than the reference cohort (3) with a median age of 64 compared to 56, respectively. There were 26 patients younger than 50 years and 115 patients 50 years of age or older. The median age was 64 (range 18-90). 88 of 141 patients completed the planned course of IMRT and concomitant TMZ with at least six cycles of adjuvant TMZ. Reasons for stopping or decreasing the dose of TMZ are listed in Table II. 53 patients stopped TMZ treatment (37.6\%). Of these, hematologic toxicity
Table I. Comparison of patient demographics at time of study between the BIDMC cohort and the reference cohort.

\begin{tabular}{lcc}
\hline Characteristic & $\begin{array}{c}\text { BIDMC } \\
\text { cohort }(\mathrm{n}=141)\end{array}$ & $\begin{array}{c}\text { Reference } \\
\text { cohort }^{\mathrm{a}}(\mathrm{n}=287)\end{array}$ \\
\hline $\begin{array}{l}\text { Age, years } \\
\text { Median }\end{array}$ & 64 & 56 \\
$\quad$ Range & $18-90$ & $19-70$ \\
Age, years, $\mathrm{n}(\%)$ & & \\
$\quad<50$ years & $26(18)$ & $90(31)$ \\
$\geq 50$ years & $115(82)$ & $197(69)$ \\
Sex, $\mathrm{n}(\%)$ & & \\
$\quad$ Male & $76(54)$ & $185(64)$ \\
Female & $65(46)$ & $102(36)$ \\
Surgery, $\mathrm{n}(\%)$ & & \\
$\quad$ Biopsy & $40(28)$ & $48(17)$ \\
Partial resection & $30(21)$ & $126(44)$ \\
$\quad$ Complete resection & $71(50)$ & $113(39)$ \\
IMRT/TMZ + cTMZ, $\mathrm{n}(\%)$ & & \\
Completed & $88(62)$ & $105(37)^{\mathrm{a}}$ \\
$\quad$ Did not complete & $53(38)$ & $182(63)$ \\
Median OS, months & 19 & 14.6 \\
Median PFS, months & 8 & 6.9 \\
\hline
\end{tabular}

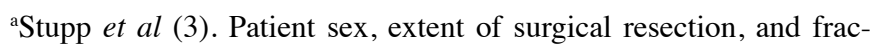
tion completed 6 weeks of IMRT/TMZ followed by six cycles of adjuvant TMZ are shown for the BIDMC cohort and the reference cohort. BIDMC, Beth Israel Deaconess Medical Center; IMRT, intensity-modulated radiation therapy; TMZ, temozolomide; cTMZ, monthly cyclic temozolomide; PFS, progression-free survival; OS, overall survival.

Table II. Reasons for discontinuation of intensity-modulated radiation therapy/TMZ and 6 cycles of adjuvant TMZ.

\begin{tabular}{lc} 
Complication & Number affected \\
\hline Disease progression & $29(20.6)$ \\
Bone marrow toxicity & $20(14.2)$ \\
Dysphagia & $1(0.7)$ \\
Cerebral hemorrhage & $1(0.7)$ \\
Cardiac arrest (unrelated) & $1(0.7)$ \\
Heart failure (unrelated) & $1(0.7)$ \\
Total & $53(37.6)$
\end{tabular}

TMZ, temozolomide.

$(n=20,14.2 \%)$ and progression of disease $(n=29,20.6 \%)$ were the two main reasons. Seventy five patients developed leukopenia, and 95 patients developed thrombocytopenia. IDH1 R132H mutation was identified in five patients, absent in 89 patients, and data was not available in 47 patients. The median OS for all 141 patients was 19 months, and the median PFS was 8 months. There were no treatment-associated deaths. 
Table III. Univariate survival analysis using Kaplan Meier analysis and the log-rank test.

\begin{tabular}{|c|c|c|c|c|}
\hline Characteristic & Median PFS, months & P-value & Median OS, months & P-value \\
\hline \multicolumn{5}{|l|}{ Age, years } \\
\hline$<50$ & 7 & \multirow[t]{2}{*}{0.262} & 22 & \multirow[t]{2}{*}{$0.006^{\mathrm{a}}$} \\
\hline$\geq 50$ & 8 & & 16 & \\
\hline \multicolumn{5}{|l|}{$\operatorname{Sex}$} \\
\hline Male & 8 & \multirow[t]{2}{*}{0.324} & 20 & \multirow[t]{2}{*}{0.742} \\
\hline Female & 8 & & 18 & \\
\hline \multicolumn{5}{|l|}{ Extent of resection } \\
\hline Total & 9 & \multirow[t]{2}{*}{0.707} & 21 & \multirow[t]{2}{*}{0.063} \\
\hline Partial, biopsy & 6 & & 14 & \\
\hline \multicolumn{5}{|l|}{ IMRT/TMZ + cTMZ } \\
\hline Completed & 9 & \multirow[t]{2}{*}{0.108} & 20 & \multirow[t]{2}{*}{$0.030^{\mathrm{a}}$} \\
\hline Did not complete & 6 & & 14 & \\
\hline \multicolumn{5}{|l|}{ Platelet count } \\
\hline No thrombocytopenia & 6 & \multirow[t]{2}{*}{0.082} & 20 & \multirow[t]{2}{*}{0.928} \\
\hline Thrombocytopenia & 9 & & 17 & \\
\hline \multicolumn{5}{|l|}{ WBC count } \\
\hline No leukopenia & 6 & \multirow[t]{2}{*}{0.088} & 14 & \multirow[t]{2}{*}{$0.008^{\mathrm{a}}$} \\
\hline Leukopenia & 9 & & 21 & \\
\hline \multicolumn{5}{|l|}{ IDH1 status } \\
\hline IDH1 wild type (89) & 8 & \multirow[t]{2}{*}{0.697} & 16 & \multirow[t]{2}{*}{0.287} \\
\hline IDH1 mutation (5) & 13 & & 23 & \\
\hline \multicolumn{5}{|c|}{ WBC, absent IDH1 R132H } \\
\hline No leukopenia (46) & 6 & \multirow[t]{2}{*}{0.116} & 13 & \multirow[t]{2}{*}{0.053} \\
\hline Leukopenia (43) & 9 & & 20 & \\
\hline \multicolumn{5}{|c|}{ WBC, present IDH1 R132H } \\
\hline No leukopenia (2) & 12 & \multirow[t]{2}{*}{0.432} & 30 & \multirow[t]{2}{*}{0.586} \\
\hline Leukopenia (3) & 13 & & 23 & \\
\hline \multicolumn{5}{|c|}{ WBC, incomplete IMRT/TMZ + cTMZ } \\
\hline No leukopenia (23) & 5 & \multirow[t]{2}{*}{0.035} & 10 & \multirow[t]{2}{*}{0.055} \\
\hline Leukopenia (30) & 8 & & 17 & \\
\hline WBC, IMRT/TMZ + cT & & & & \\
\hline No Leukopenia (43) & 7 & 0.532 & 16 & 0.048 \\
\hline Leukopenia (45) & 9 & & 24 & \\
\hline
\end{tabular}

${ }^{\mathrm{a}} \mathrm{P}<0.05$. For the categories of IDH1 status, WBC with absent IDH1 R132H, WBC with present IDH1 R132H, WBC with incomplete IMRT/TMZ + cTMZ, and WBC with IMRT/TMZ + cTMZ, the values in brackets indicate the number of patients in each subcategory. Patient age, sex, extent of resection, fraction completed 6 weeks of IMRT/TMZ followed by six cycles of adjuvant TMZ, platelet count, WBC count and IDH R132H status were analyzed, PFS, OS and corresponding P-values are shown. Kaplan-Meier analysis with log rank tests was performed using statistical computing language and survival analysis libraries ( $\mathrm{R}$ version 4.0.2; R Foundation). IMRT/TMZ, intensity-modulated radiation therapy/temozolomide; PFS, progression-free survival; OS, overall survival; WBC, white blood cell count; IDH1, isocitrate dehydrogenase 1 .

Correlation of leukopenia with survival. Younger patient age ( $<50$ years old) and development of leukopenia correlated with increased OS. Median OS for patients younger than 50 years old was 22 months compared to 16 months in patients 50 years of age and older $(\mathrm{P}=0.006)$. There is a significant increase in OS in patients who develop leukopenia $(\mathrm{P}=0.008)$. In patients with leukopenia, median OS was 21 months compared to 14 months in those without leukopenia ( $\mathrm{P}=0.008$; Table III). Subgroup analysis showed that leukopenia in patients with IDH1 R132H mutation does not correlate with increased OS, although the numbers are low. While sex was not a significant determinant of outcomes, completion of IMRT/TMZ with cyclic TMZ was associated with longer OS. Patients who had total resections had a median OS of 21 months compared to 14 months in patients with partial resections or biopsies $(\mathrm{P}=0.063)$. Those who completed the full course of IMRT/TMZ with six cycles of adjuvant TMZ had a median OS of 20 months compared to patients unable to complete the regimen (OS 14 months; 
Table IV. Survival analysis of leukopenia grade using Kaplan Meier analysis and log-rank test.

\begin{tabular}{|c|c|c|c|c|c|}
\hline Grade & Patients, n (\%) & Median PFS, months & P-value & Median OS, months & P-value \\
\hline 0 & $66(47)$ & 6 & & 14 & \\
\hline 1 & $41(29)$ & 8 & 0.918 & 20 & 0.069 \\
\hline 2 & $18(13)$ & 11 & $0.033^{\mathrm{a}}$ & 29 & $0.024^{\mathrm{a}}$ \\
\hline 3 & $9(6)$ & 10 & $0.047^{\mathrm{a}}$ & 15 & 0.278 \\
\hline 4 & $7(5)$ & 11 & 0.544 & 25 & 0.417 \\
\hline
\end{tabular}

${ }^{a} \mathrm{P}<0.05$. PFS and OS are shown with patient numbers for each grade of leukopenia. Corresponding P-values are indicated for the leukopenia grade observed, relative to grade 0 . Kaplan-Meier analysis with log rank tests was performed using statistical computing language and survival analysis libraries (R version 4.0.2; R Foundation). PFS, progression-free survival; OS, overall survival.

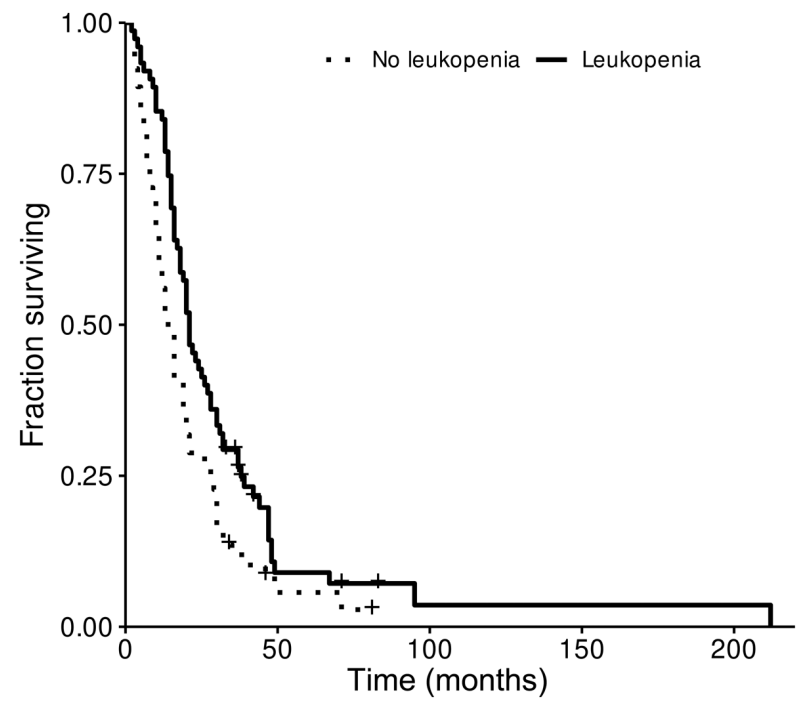

Figure 1. Survival curve of patients who developed leukopenia in the setting of TMZ exposure. OS curve for patients who developed leukopenia after exposure to TMZ. Median OS was 21 months vs. 14 months. OS, overall survival; TMZ, temozolomide.

$\mathrm{P}=0.030$; Table III). There was no significant difference in median PFS in patients who developed leukopenia $(\mathrm{P}=0.088)$. There was no significant difference in median OS or PFS in patients who developed thrombocytopenia $(\mathrm{P}=0.082$ and $\mathrm{P}=0.928$, respectively).

Correlation of leukopenia grade with survival. Kaplan-Meier survival representation of the correlation of leukopenia with OS is shown in Fig. 1. Of the patients who developed leukopenia, those who developed grade 2 leukopenia had a median OS of 28.5 months compared to 14 months in patients who maintained a normal white blood cell count $(\mathrm{P}=0.024)$. When assessing OS in patients with grades 1, 3, and 4 leukopenia compared to patients who did not develop leukopenia, there was no significant difference ( $\mathrm{P}>0.05$; Table IV). Multivariate analysis using Cox proportional hazards model revealed that leukopenia grade was associated with longer OS after taking into account other known predictors of survival such as age, extent of surgery, and completed standard TMZ ( $\mathrm{P}=0.00205$, likelihood ratio test $\mathrm{P}=4.41 \mathrm{e}-09^{*}$; Table $\mathrm{V}$ ). In this analysis, age, extent of surgery and completion of standard TMZ therapy were also highly associated with longer OS.

\section{Discussion}

In this study, we demonstrate that leukopenia in the setting of TMZ exposure is an apparent predictor of improved OS in GBM patients. Compared to a landmark reference study (3), our cohort was older (median age 56 compared to 64, respectively). The percentage of patients able to complete six weeks of IMRT/TMZ followed by six cycles of TMZ was similar, though the reasons for discontinuation differed. Whereas $14.2 \%$ of our patients stopped treatment or had treatment held for hematologic toxicity, $17 \%$ of patients in the reference cohort stopped treatment for bone marrow suppression. Thirty-nine percent of patients in the reference cohort discontinued treatment due to disease progression compared to $20.6 \%$ of our patients. The reference cohort had a median OS of 14.6 months and a median PFS of 6.9 months while our cohort had a median OS of 19 months and a median PFS of 8 months. Our data is limited in that we are unable to determine the precise dose of corticosteroid exposure at the time of TMZ treatment. A follow-up study to the landmark reference study showed that corticosteroids can compromise survival in GBM patients, and $67 \%$ of patients who were part of the reference cohort were on corticosteroid therapy at the time of the study (14).

There have been three prior publications suggesting that myelosuppression in the form of thrombocytopenia or neutropenia is associated with better OS in patients who have had surgery, radiation, and TMZ. The initial study found that during six weeks of radiation with concomitant TMZ in 84 patients, a decrease in platelet count compared to baseline correlated with prolonged OS whereas changes in lymphocyte count showed no such correlation (13). Lymphocyte count was chosen as a general marker for myelosuppression as this was more common than neutropenia. Neutrophil count and general leukopenia were not evaluated. The second study reviewed changes in neutrophil count during the course of standard treatment for correlation with survival (12). It was reported that MGMT promoter methylation, wild type EGFR status, younger age at diagnosis, and treatment-induced decreases in white blood cell (WBC) count in 86 patients who completed the full six-week course of IMRT/TMZ followed by six cycles of adjuvant TMZ had increased median OS. IDH1 R132H mutation and MGMT promoter methylation did not correlate with changes in WBC counts. In our study, patients who were unable to complete the standard treatment of six weeks IMRT/TMZ with six cycles of adjuvant TMZ 
Table V. Multivariate analysis using Cox proportional hazards model.

\begin{tabular}{llc}
\hline Variable & P-value & Hazard ratio (95\% CI) \\
\hline Age & $5.92 \times 10-6^{\mathrm{a}}$ & $1.03(1.02-1.05)$ \\
Sex & 0.94627 & $0.99(0.68-1.43)$ \\
Extent of surgery & $0.00106^{\mathrm{a}}$ & $0.71(0.57-0.87)$ \\
Leukopenia grade & $0.00205^{\mathrm{a}}$ & $0.77(0.65-0.91)$ \\
TMZ completed & $0.00252^{\mathrm{a}}$ & $1.80(1.23-2.64)$ \\
\hline
\end{tabular}

${ }^{\mathrm{a}} \mathrm{P}<0.05$. Patient age, sex, extent of surgery and leukopenia grade were analyzed for association with overall survival, and P-values are shown for each individual variable. Likelihood ratio test $\mathrm{P}=4.41 \times 10^{-9}$. Cox multivariate proportional hazards analysis was performed using statistical computing language and survival analysis libraries ( $\mathrm{R}$ version 4.0.2; $\mathrm{R}$ Foundation; 'survival' library version 3.2-3; 'survminer' library version 0.4 .8 ). TMZ, temozolomide.

were not excluded. Instead, all patients who were exposed to TMZ were included to assess bone marrow suppression and survival.

The third study assessed the change in neutrophil count in 50 patients with absent IDH1 R132H mutation during the initial six weeks of IMRT/TMZ only (11). They found that patients with greater than or equal to a $40 \%$ decrease in neutrophils showed significantly longer OS than those with less than a $40 \%$ decrease. They also report that extent of resection, MGMT status, decreased rate of WBC count, and decreased rate of platelet count were associated with increased median OS by univariate analysis. After adjusting for various factors, however, only the decrease in neutrophils remained significant. In our subset of IDH1 R132H negative patients, OS between patients who developed leukopenia and those who did not with any TMZ exposure approached statistical significance $(\mathrm{P}=0.05)$. Our patients with IDH1 R132H mutation did not have increased OS, probably the result of the small sample size $(n=5)$. Another prior study evaluated platelet count as a marker of outcomes in this setting and found that an increase in platelet count during chemoradiation was associated with poor outcome (15). Platelet count, however, is unlikely to be useful as biomarker of bone marrow suppression owing to multiple factors that are unrelated to megakaryocyte activity such as consumption rate and acute-phase induction.

The association of leukopenia with OS is not fully understood. We hypothesize that TMZ causes marrow suppression depending on MGMT repair activity, which in turn depends on factors like TMZ clearance, MGMT promoter methylation, MGMT protein expression, and polymorphisms and mutations affecting MGMT enzyme stability and activity. Current dosing practices do not take into account such individual differences, resulting in a large proportion of patients experiencing dose-limiting toxicity due to slow clearance and low MGMT repair activity. It can be assumed that some patients are relatively under-dosed with TMZ due to fast TMZ clearance or high MGMT repair activity (16). This raises the possibility that there are patients who are relatively under-dosed with current standard-of-care treatment and would do better with higher doses of TMZ, perhaps on a protocol targeting brief nadirs of grade 2 leukopenia.

The limitations of this study include its observational design and performance in a single-institution involving a relatively small number of patients. Important clinical data such as corticosteroid use and MGMT methylation status were not available. This study does however confirm earlier reports of the association between bone marrow suppression and improved OS in GBM. For better understanding of this phenomenon, further studies such as a randomized controlled TMZ dose escalation trial or a two-tier dose stratification trial based on MGMT promoter methylation is needed.

In conclusion, a possible interpretation of these results is that grade 2 leukopenia is a biomarker of adequate TMZ dosing in a population with diverse DNA repair function, which in turn may be the consequence of variable MGMT activity. A prospective dose escalation trial is necessary to determine if treatment-induced leukopenia is beneficial for all patients receiving $\mathrm{TMZ}$.

\section{Acknowledgements}

The authors would like to thank Mrs. Julianne Bloom, RN and Ms. Loretta Barron, NP (Brain Tumor Clinic, Department of Neurology, Beth Israel Deaconess Hospital, Boston, USA) for coordinating the care of our patients.

\section{Funding}

No funding was received.

\section{Availability of data and materials}

The datasets used and/or analyzed during the current study are available from the corresponding author on reasonable request.

\section{Authors' contributions}

KGH, EJU and ETW conceived and designed the study. $\mathrm{KGH}$ drafted the article. ENU and $\mathrm{KGH}$ performed statistical analysis. KGH, EJU and ETW critically revised the article. $\mathrm{KGH}, \mathrm{EJU}, \mathrm{ETW}$ and ENU reviewed the submitted version of the manuscript. All authors read and approved the final manuscript.

\section{Ethics approval and consent to participate}

Ethical approval was obtained from the institutional review board of the Dana Farber Harvard Cancer Center. The Institutional Review Board waived the requirement for informed consent due to retrospective design. Once the clinical data was collected, all identifiers were removed.

\section{Patient consent for publication}

Not applicable.

\section{Competing interests}

The authors declare that they have no competing interests. 


\section{References}

1. Gilbert MR, Wang M, Aldape KD, Stupp R, Hegi ME, Jaeckle KA, Armstrong TS, Wefel JS, Won M, Blumenthal DT, et al: Dose-dense temozolomide for newly diagnosed glioblastoma: A randomized phase III clinical trial. J Clin Oncol 31: 4085-4091, 2013.

2. Stupp R, Hegi ME, Mason WP, van den Bent MJ, Taphoorn MJ, Janzer RC, Ludwin SK, Allgeier A, Fisher B, Belanger K, et al: Effects of radiotherapy with concomitant and adjuvant temozolomide versus radiotherapy alone on survival in glioblastoma in a randomised phase III study: 5-year analysis of the EORTC-NCIC trial. Lancet Oncol 10: 459-466, 2009.

3. Stupp R, Mason WP, van den Bent MJ, Weller M, Fisher B, Taphoorn MJ, Belanger $\mathrm{K}$, Brandes AA, Marosi C, Bogdahn U, et al: Radiotherapy plus concomitant and adjuvant temozolomide for glioblastoma. N Engl J Med 352: 987-996, 2005 .

4. Tolcher AW, Gerson SL, Denis L, Geyer C, Hammond LA, Patnaik A, Goetz AD, Schwartz G, Edwards T, Reyderman L, et al: Marked inactivation of O6-alkylguanine-DNA alkyltransferase activity with protracted temozolomide schedules. Br J Cancer 88: 1004-1011, 2003.

5. Knizhnik AV, Roos WP, Nikolova T, Quiors S, Tomaszowski K, Christmann M and Kaina B: Survival and death strategies in glioma cells: Autophagy, senescence and apoptosis triggered by a single type of temozolomide-induced DNA damage. PLoS One 8: e55665, 2013.

6. Gerson SL, Phillips W, Kastan M, Dumenco LL and Donovan C: Human $\mathrm{CD} 4^{+}$hematopoietic progenitors have low, cytokine-unresponsive O6-alkylguanine-DNA alkyltransferase and are sensitive to O6-benzylguanine plus BCNU. Blood 88: 1649-1655, 1996.

7. Newlands ES, Stevens MF, Wedge SR, Wheelhouse RT and Brock C: Temozolomide: A review of its discovery, chemical properties, pre-clinical development and clinical trials. Cancer Treat Rev 23: 35-61, 1997.

8. Abola MV, Prasad V and Jena AB: Association between treatment toxicity and outcomes in oncology clinical trials. Ann Oncol 25: 2284-2289, 2014.
9. Di Maio M, Gridelli C, Gallo C, Shepherd F, Piantedosi FV, Cigolari S, Manzione L, Illiano A, Barbera S, Robbiati SF, et al: Chemotherapy-induced neutropenia and treatment efficacy in advanced non-small-cell lung cancer: A pooled analysis of three randomised trials. Lancel Oncol 6: 669-677, 2005.

10. Fontein DB, Seynaeve C, Hadji P, Hille ET, van de Water W, Putter H, Kranenbarg EM, Hasenburg A, Paridaens RJ, Vannetzel JM, et al: Specific adverse events predict survival benefit in patients treated with tamoxifen or aromatase inhibitors: An international tamoxifen exemestane adjuvant multinational trial analysis. J Clin Oncol 31: 2257-2264, 2013.

11. Saito T, Sugiyama K, Hama S, Yamasaki F, Takayasu T, Nosaka R, Muragaki Y, Kawamata T and Kurisu K: Prognostic importance of temozolomide-induced neutropenia in glioblastoma, IDH-wildtype patients. Neurosurg Rev 41: 621-628, 2018.

12. Vaios EJ, Nahed BV, Muzikansky A, Fathi AT and Dietrich J: Bone marrow response as a potential biomarker of outcomes in glioblastoma patient. J Neurosurg 127: 132-138, 2017.

13. Williams M, Liu ZW, Woolf D, Hargreaves S, Michalarea V, Menashy R, Kooner I and Wilson E: Change in platelet levels during radiotherapy with concurrent and adjuvant temozolomide for the treatment of glioblastoma: A novel prognostic factor for survival. J Cancer Res Clin Oncol 138: 1683-1688, 2012.

14. Pitter K, Tamagno I, Alikhanyan K, Hosni-Ahmen A, Pattwell SS Donnola S, Dai C, Ozawa T, Chang M, Chan TA, et al: Corticosteroids compromise survival in glioblastoma. Brain 139: 1458-1471, 2016.

15. Boonyawan K, Hess KR, Yang J, Long L, Wang Q, Ezhilarasan R, Auia A, Alfaro-Munoz KD, de Groot JF, Bhat KP and Sulman EP: A relative increase in circulating platelets following chemoradiation predicts for poor survival of patients with glioblastoma. Oncotarget 8: 90488-90495, 2017.

16. Armstrong TS, Cao Y, Scheurer ME, Vera-Bolaños E, Manning R, Okcu MF, Bondy M, Zhou R and Gilbert MR: Risk analysis of severe myelotoxicity with temozolomide: The effects of clinical and genetic factors. Neuro Oncol 11: 825-832, 2009.

This work is licensed under a Creative Commons Attribution-NonCommercial-NoDerivatives 4.0 International (CC BY-NC-ND 4.0) License. 